\title{
Comparison of surgically induced astigmatism following different glaucoma operations
}

This article was published in the following Dove Press journal: Clinical Ophthalmology

\section{Masaki Tanito \\ Yukari Matsuzaki \\ Yoshifumi Ikeda \\ Etsuko Fujihara}

Division of Ophthalmology, Matsue Red Cross Hospital, Matsue, Japan
Correspondence: Masaki Tanito Division of Ophthalmology, Matsue Red Cross Hospital, 200 Horo-machi, Matsue, Shimane, 690-8506, Japan

Tel +8I 8522421 II

Fax +8I 852 3। 9783

Email tanito-oph@umin.ac.jp
Aim: To compare surgically induced astigmatism (SIA) among glaucomatous eyes treated with trabeculectomy (LEC), EX-PRESS ${ }^{\circledR}$ shunt (EXP), ab externo trabeculotomy (exLOT), or microhook ab interno trabeculotomy ( $\mu$ LOT).

Subjects and methods: Eighty right eyes of 80 subjects who underwent LEC $(n=20)$, EXP $(n=20)$, exLOT $(n=20)$, or $\mu$ LOT $(n=20)$ were included. The dataset including the best-corrected visual acuity (BCVA), intraocular pressure (IOP), and keratometry recordings preoperatively and 3 months postoperatively was collected by chart review. The means of the vector magnitude, vector meridian, and arithmetic magnitude of the preoperative and postoperative astigmatism and SIA were calculated. The correlations among the SIA magnitude, postoperative BCVA, and IOP were assessed.

Results: The mean astigmatic arithmetic magnitudes did not differ significantly $(P=0.0732)$ preoperatively among the four groups, but the magnitude was significantly $(P=0.0002)$ greater in the LEC group than the other groups postoperatively. The mean SIA vectors were calculated to be $1.01 \mathrm{D}$ at $56^{\circ}, 0.62 \mathrm{D}$ at $74^{\circ}, 0.23 \mathrm{D}$ at $112^{\circ}$, and $0.12 \mathrm{D}$ at $97^{\circ}$ for the $\mathrm{LEC}, \mathrm{EXP}$, exLOT, and $\mu$ LOT groups, respectively. The mean SIA arithmetic magnitudes were significantly $(P<0.0001)$ greater in the LEC group than the other groups. Three months postoperatively, the SIA magnitude was correlated positively with the logarithm of the minimum angle of resolution (logMAR) BCVA ( $r=0.3538)$ and negatively with the IOP $(r=-0.3265)$; the logMAR BCVA was correlated negatively with the IOP $(r=-0.3105)$.

Conclusion: EXP, exLOT, and $\mu$ LOT induce less corneal astigmatism than LEC in the early postoperative period.

Keywords: SIA, trabeculectomy, EX-PRESS shunt, ab externo trabeculotomy, microhook ab interno trabeculotomy, MIGS

\section{Introduction}

Trabeculectomy (LEC) remains the standard surgical procedure for glaucoma when medical and laser treatments fail to control the intraocular pressure (IOP). The astigmatic changes after LEC can lead to decreased visual acuity (VA) ${ }^{1}$ and might be distress for patients. As a result, a number of investigators have studied corneal refractive changes or astigmatism induced by LEC. ${ }^{1-10}$ However, few studies have reported the impact of surgically induced alterations in keratometry resulting from other glaucoma surgeries such as the EX-PRESS ${ }^{\circledR}$ shunt (EXP) (Alcon Japan, Tokyo, Japan), ab externo trabeculotomy (exLOT), and minimally invasive glaucoma surgery (MIGS).

We compared the surgically induced astigmatism (SIA) among four glaucoma surgeries, that is, conventional (LEC) and novel (EXP) filtration surgeries, conventional nonfiltration surgery (exLOT), and novel MIGS (ie, microhook ab interno trabeculotomy $[\mu \mathrm{LOT}])^{11,12}$ 


\section{Subjects and methods}

This retrospective study included 80 right eyes of 80 consecutive subjects who underwent LEC $(n=20), \operatorname{EXP}(n=20)$, $\operatorname{exLOT}(\mathrm{n}=20)$, or $\mu$ LOT $(\mathrm{n}=20)$ at Matsue Red Cross Hospital between April 2014 and July 2016. The patients who met the inclusion criteria were selected from our division's glaucoma database in a chronological order of surgical date to include 20 eyes in each of the surgical groups. The criteria common for all four surgical groups included the following: the availability of data from the right eye; the patients underwent one of the four glaucoma surgeries performed by the same surgeon (MT); the patients did not undergo a simultaneous cataract surgery or other procedures; and the recorded data included the best-corrected visual acuity (BCVA), IOP, and keratometry recordings obtained preoperatively and 3 months (2-4 months) postoperatively. The BCVA measured using a decimal VA chart was converted to the logarithm of the minimum angle of resolution (logMAR) VA. The IOP was measured by Goldmann applanation tonometry. The keratometry was recorded at the central $3 \mathrm{~mm}$ diameter by autorefractor keratometer (RC5000; Tomey, Nagoya, Japan).

The current study was conducted as part of a study protocol titled "Epidemiologic study in ocular morphology and function", approved by the institutional review board of Matsue Red Cross Hospital; the study adhered to the tenets of the Declaration of Helsinki. The ethics committee waived the requirement for the patients' informed consent regarding the use of their medical record data in accordance with the regulations of the Japanese Guidelines for Epidemiologic Study issued by the Japanese Government, and instead, the protocol was posted at the outpatient clinic to notify participants of the study. Only the anonymized data were used in the statistical analyses.

\section{Surgical procedure}

All surgeries were performed under miosis induced by topical 1\% pilocarpine (Sanpilo; Santen Pharmaceutical, Osaka, Japan) and standard sub-Tenon anesthesia using $2 \%$ lidocaine. At the end of surgery, $1.65 \mathrm{mg}$ of dexamethasone sodium phosphate (Decadron; Aspen Japan, Tokyo, Japan) was injected subconjunctivally and $0.3 \%$ ofloxacin ointment (Tarivid; Santen Pharmaceutical) was applied. Finally, $1.5 \%$ levofloxacin (Nipro, Osaka, Japan) and $0.1 \%$ betamethasone (Sanbetason; Santen Pharmaceutical) were applied topically four times daily for 3-4 weeks postoperatively in all cases.

\section{LEC}

Surgery was performed in the superonasal quadrant. After creation of a conjunctival peritomy of less than one quadrant, a half-thickness $\sim 3-4 \times \sim 3-4 \mathrm{~mm}^{2}$ first scleral flap was created. After the first flap was dissected, $0.04 \%$ mitomycin $\mathrm{C}$ was applied for 3 minutes followed by rinsing with $100 \mathrm{~mL}$ of balanced salt solution. Under the first flap, a second flap of fourfifth thickness was created inside the scleral bed of the first flap, and then the trabecular tissue was excised en bloc in the second scleral flap. After a peripheral iridectomy was performed, the first scleral flap was closed with four interrupted 10-0 nylon sutures (Mani, Utsunomiya, Japan). The conjunctiva was readapted with 10-0 absorbable sutures (Vicryl; Johnson \& Johnson, New Brunswick, NJ, USA). Depending on the IOP level and bleb formation, laser suture lysis was started 3 days postoperatively and ended by 1 month postoperatively. Typically, suture lysis was performed when the IOP exceeded $12 \mathrm{mmHg}$.

\section{EXP}

Surgery was performed in the superonasal quadrant. ${ }^{13}$ After a conjunctival peritomy was created, a halfthickness $\sim 3-4 \times \sim 3-4 \mathrm{~mm}^{2}$ scleral flap was created. A model P-50 EX-PRESS shunt was inserted under the scleral flap into the anterior chamber through a scleral tunnel created using a 25 -gauge needle. Before shunt insertion, $0.04 \%$ mitomycin C was applied for 3 minutes followed by rinsing with $100 \mathrm{~mL}$ of balanced salt solution. The scleral flap then was closed with three interrupted 10-0 nylon sutures (Mani, Utsunomiya, Japan). The conjunctiva was readapted with 10-0 absorbable sutures (Vicryl, Johnson \& Johnson). Laser suture lysis was performed as described for the LEC procedure.

\section{exLOT}

Surgery was performed in the inferonasal quadrant. ${ }^{14}$ After the conjunctival peritomy was created, a half-thickness $4 \times 4 \mathrm{~mm}$ triangular first scleral flap was created. Under that flap, a second flap of four-fifth thickness was created inside the scleral bed of the first flap. Schlemm's canal was then exposed, and the outer wall of Schlemm's canal was excised en bloc in the second scleral flap. Metal trabeculotomes (M-80A-15; Inami, Tokyo, Japan) were inserted into Schlemm's canal from both openings at the sidewalls of the scleral lake and rotated into the anterior chamber to incise the inner wall of Schlemm's canal and the trabecular meshwork. The trabeculotomy extended about one-third of the circumference. The first scleral flap was closed with three interrupted 10-0 nylon sutures (Mani). The conjunctiva was readapted with 10-0 absorbable sutures (Vicryl; Johnson \& Johnson).

\section{$\mu \mathrm{LOT}$}

The surgery was performed through two corneal side ports. ${ }^{11,12}$ A spatula-shaped microhook designed specifically 
for use during $\mu \mathrm{LOT}$ was used (M-2215; Inami). Viscoelastic material (1\% sodium hyaluronate; Opegan Hi; Santen Pharmaceutical) was injected into the anterior chamber through the clear corneal ports created using a 20-gauge micro-vitreoretinal knife (Mani) at the 2-3 and 9-10 o'clock positions. A microhook was inserted into the anterior chamber through the corneal port using a Swan-Jacob gonioprism lens (Ocular Instruments, Bellevue, WA, USA) to observe the angle opposite to the corneal port. The tip of the microhook was then inserted into Schlemm's canal and moved circumferentially to incise the inner wall of Schlemm's canal and trabecular meshwork over 3 clock hours. Using the same procedure, trabeculotomy was performed in the opposite angle using a microhook inserted through the other corneal port. The trabeculotomy extended about two-thirds of the circumference. After the viscoelastic material was aspirated bimanually, the corneal ports were closed by corneal stromal hydration.

\section{Calculation of astigmatism parameters}

The preoperative and postoperative astigmatism levels and SIA were calculated from the keratometric values obtained preoperatively and 3 months postoperatively using the SIA Calculator Version 2.1 developed by Drs Saurabh Sawhey and Aashima Aggarwal (http://www.insighteyeclinic.in/SIA calculator.php $)^{15}$ based on the vector analysis algorithm. ${ }^{16,17}$ Before the analysis, all the keratometric astigmatism values were converted into plus-cylinder format. The means of the vector magnitude, vector meridian, and arithmetic magnitude of the preoperative and postoperative astigmatism and SIA values were obtained in each surgical group. The distributions of the SIA in each surgical group were visualized on a scatter plot using the Astig PLOT application (http:// www.edmondoborasio.com/Doctor Edmondo Borasio/ Astig PLOT.html).

\section{Statistical analysis}

The age, BCVA, IOP, and three astigmatism parameters were compared among the four surgical groups by one-way analysis of variance (ANOVA) followed by a comparison between each pair of groups using the post hoc Student's $t$-test. In ANOVA, $P<0.05$ was considered significant. In the post hoc test, based on Bonferroni's method to correct multiple comparisons, $P<0.0083$ and $P<0.0017$ were considered significant at the probability levels of $5 \%$ and $1 \%$, respectively. Sex was compared among the four surgical groups using the chi-square test. The possible correlations among the SIA magnitude, postoperative BCVA, and IOP were assessed using the Spearman's rank correlation test; to correct multiple comparisons, $P<0.00167$ and $P<0.0033$ were considered significant at the probability levels of $5 \%$ and $1 \%$, respectively. All continuous data were expressed as the means \pm standard deviations. All statistical analyses were performed using the JMP version 11.0 statistical software (SAS Institute, Inc., Cary, NC, USA).

\section{Results}

The demographic data obtained from subjects included patient age, sex, glaucoma type, preoperative and postoperative BCVA, and IOP (Table 1). The preoperative and postoperative BCVAs were significantly worse in the LEC group compared with the other three groups. The postoperative IOP was significantly $(P=0.0016)$ lower in the LEC and EXP groups than in the $\mu$ LOT group.

The astigmatism parameters calculated are summarized in Table 2 and Figure 1. The arithmetic magnitudes of astigmatism did not differ significantly ( $P=0.0732$, ANOVA) among the four groups preoperatively; the arithmetic magnitude of astigmatism was significantly ( $P=0.0002$, ANOVA) higher in the LEC group than in the other three groups postoperatively. The mean SIA vectors were calculated to be $1.01 \mathrm{D}, 0.62 \mathrm{D}, 0.23 \mathrm{D}$, and $0.12 \mathrm{D}$ for the LEC, EXP, exLOT, and $\mu$ LOT groups, respectively. The SIA was on the superonasal to inferotemporal meridians in the LEC and EXP groups and on the superotemporal to inferonasal meridians in the LOT group. The arithmetic magnitude of astigmatism was significantly ( $P<0.0001$, ANOVA) higher in the LEC group than in the other groups. The SIA magnitudes and meridians varied among the patients in the LEC group and were more uniform in the exLOT and $\mu$ LOT groups (Figure 1).

The SIA magnitude was correlated positively with the $\log$ MAR BCVA $(r=0.3538)$ and negatively with the IOP $(r=-0.3265)$, and the logMAR BCVA was correlated negatively with the IOP $(r=-0.3105)$ at 3 months postoperatively (Table 3).

\section{Discussion}

Few reports have compared the SIA among different glaucoma surgeries ${ }^{7,8}$ or between modified techniques of the same glaucoma surgery. ${ }^{5}$ In the current study, the SIA magnitude in the LEC group did not differ markedly from the SIA values obtained from the previously reported vector analysis at 1-6 months after LEC. ${ }^{7,10}$ Thus, our results indicated that the EXP, exLOT, and $\mu \mathrm{LOT}$ surgeries induce less astigmatism than LEC.

We observed oblique astigmatism in three surgical groups; the SIA meridians were directed to the location of the scleral flaps (ie, superonasally in the LEC and EXP groups and inferonasally in the exLOT group). After LEC, 
Table I Subject characteristics

\begin{tabular}{|c|c|c|c|c|c|}
\hline Parameters & LEC & EXP & exLOT & $\mu \mathrm{LOT}$ & $P$-value \\
\hline Eyes/subjects, $n / n$ & $20 / 20$ & $20 / 20$ & $20 / 20$ & $20 / 20$ & \\
\hline \multicolumn{5}{|l|}{ Age (years) } & $0.9345^{\#}$ \\
\hline Mean \pm SD & $65.1 \pm 12.3$ & $66.8 \pm 10.7$ & $65.6 \pm 19.9$ & $68.0 \pm 16.1$ & \\
\hline $95 \% \mathrm{Cl}$ & $59.3-70.9$ & $61.8-71.8$ & $56.3-74.9$ & $60.4-75.5$ & \\
\hline \multicolumn{5}{|l|}{ Sex, n (\%) } & $0.5682^{\S}$ \\
\hline Male & II (55) & $10(50)$ & $7(35)$ & $8(40)$ & \\
\hline Female & $9(45)$ & $10(50)$ & $13(65)$ & $12(60)$ & \\
\hline \multicolumn{6}{|c|}{ Glaucoma type, n (\%) } \\
\hline POAG & $14(70)$ & $16(80)$ & $10(50)$ & $12(60)$ & \\
\hline SOAG & $3(15)$ & $3(15)$ & $7(35)$ & $3(15)$ & \\
\hline PACG & I (5) & I (5) & $2(15)$ & $4(20)$ & \\
\hline SACG & $2(10)$ & $0(0)$ & $0(0)$ & I (5) & \\
\hline \multicolumn{5}{|c|}{ Preoperative BCVA (logMAR) } & $0.0054^{\#}$ \\
\hline Mean \pm SD & $0.33 \pm 0.58$ & $0.02 \pm 0.17$ & $0.00 \pm 0.10$ & $0.08 \pm 0.24$ & \\
\hline $95 \% \mathrm{Cl}$ & $0.07-0.60$ & -0.06 to 0.1 & -0.05 to 0.04 & -0.03 to 0.19 & \\
\hline \multicolumn{6}{|l|}{$P$-values ${ }^{\ddagger}$} \\
\hline Against EXP & $0.0028 *$ & - & - & - & \\
\hline Against exLOT & $0.0016 * *$ & 0.8551 & - & - & \\
\hline Against $\mu \mathrm{LOT}$ & 0.0157 & 0.5398 & 0.4267 & - & \\
\hline \multicolumn{5}{|c|}{ Postoperative 3 months BCVA (logMAR) } & $0.0003^{\#}$ \\
\hline Mean \pm SD & $0.43 \pm 0.50$ & $0.09 \pm 0.27$ & $0.03 \pm 0.14$ & $0.06 \pm 0.21$ & \\
\hline $95 \% \mathrm{Cl}$ & $0.20-0.66$ & -0.04 to 0.21 & -0.04 to 0.10 & -0.03 to 0.16 & \\
\hline \multicolumn{6}{|l|}{$P$-values ${ }^{\ddagger}$} \\
\hline Against EXP & $0.0008^{* *}$ & - & - & - & \\
\hline Against exLOT & $0.000 I^{* *}$ & 0.5644 & - & - & \\
\hline Against $\mu \mathrm{LOT}$ & $0.0004^{* *}$ & 0.8337 & 0.7137 & - & \\
\hline \multicolumn{5}{|c|}{ Preoperative IOP $(\mathrm{mmHg})$} & $0.1463^{\#}$ \\
\hline Mean \pm SD & $24.8 \pm 10.1$ & $19.4 \pm 7.1$ & $22.5 \pm 11.4$ & $19.2 \pm 5.8$ & \\
\hline $95 \% \mathrm{Cl}$ & $20.1-29.5$ & $16.1-22.7$ & |7.2-27.8 & $16.4-21.9$ & \\
\hline \multicolumn{5}{|c|}{ Postoperative 3 months IOP (mmHg) } & $0.0016^{\#}$ \\
\hline Mean \pm SD & $10.6 \pm 5.5$ & $9.4 \pm 3.7$ & $12.9 \pm 3.4$ & $14.3 \pm 3.8$ & \\
\hline $95 \% \mathrm{Cl}$ & $8.0-13.1$ & $7.7-11.1$ & $|\mathrm{I} .3-| 4.4$ & $12.5-16.1$ & \\
\hline \multicolumn{6}{|l|}{$P$-values ${ }^{\ddagger}$} \\
\hline Against EXP & 0.3871 & - & - & - & \\
\hline Against exLOT & 0.0860 & 0.0109 & - & - & \\
\hline Against $\mu \mathrm{LOT}$ & $0.0058 *$ & $0.0004 * *$ & 0.2762 & - & \\
\hline
\end{tabular}

Notes: $P$-values are calculated among four glaucoma surgeries by one-way analysis of variance $\left({ }^{\#}\right)$ followed by comparison between each pair of groups using the post hoc Student $t$-test $\left(^{\ddagger}\right)$ for the continuous variables, and by the chi-square test (\$) for the categorical variables. In the post hoc test, based on Bonferroni's method to correct multiple comparisons, $P<0.0083$ and $P<0.0017$ are considered significant at the probability levels of $5 \%(*)$ and $1 \%(* *)$, respectively.

Abbreviations: LEC, trabeculectomy; EXP, EX-PRESS ${ }^{\circledast}$ shunt; exLOT, ab externo trabeculotomy; $\mu$ LOT, microhook ab interno trabeculotomy; SD, standard deviation; $\mathrm{Cl}$, confidence interval; POAG, primary open-angle glaucoma; SOAG, secondary open-angle glaucoma; PACG, primary angle-closure glaucoma; SACG, secondary angleclosure glaucoma; BCVA, best-corrected visual acuity; logMAR, logarithm of the minimum angle of resolution; IOP, intraocular pressure.

most authors have observed a with-the-rule (WTR) shift in the corneal astigmatism. ${ }^{2-4,9,10}$ In those studies, a scleral flap was created at the superior limbus. The evidence suggested that the scleral flap itself affects the direction of the SIA meridian after the glaucoma surgeries. The reason for astigmatism induction after LEC remains unclear, but a couple of mechanisms have been postulated. First, the astigmatism shift might be due to a surgically induced gape around the scleral flap rather than the number of flap sutures. ${ }^{1}$ A second possibility is that when tissue is removed from under the scleral flap and then is sutured back, the flap approaches the corneal edge and allows the unsupported corneal edge of the LEC opening to sink slightly. ${ }^{2}$ The first mechanism should be associated with the procedures in which scleral flaps are created. However, because no tissue was removed, the second mechanism was absent in the EXP surgery. Thus, absence of the second mechanism can explain the lower SIA in the EXP procedure compared with the LEC in this study.

The current study found a significant association between higher SIA and lower IOP level. Delbeke et al reported a significant ( $r=-0.49, P=0.001$ ) correlation between the magnitude of astigmatism and the IOP level, 1 month after LEC, 
Table 2 Comparison of astigmatism parameters among the surgical groups

\begin{tabular}{|c|c|c|c|c|c|}
\hline Parameters & LEC & EXP & exLOT & $\mu \mathrm{LOT}$ & P-value \\
\hline \multicolumn{5}{|l|}{ Preoperative astigmatism } & 0.0732 \\
\hline Vector magnitude (D) & $0.22 \pm 1.30$ & $0.18 \pm 1.05$ & $0.37 \pm 0.87$ & $0.42 \pm 1.69$ & \\
\hline Vector meridian (degrees) & $38 \pm 10$ & $44 \pm 11$ & $21 \pm 17$ & $17 \pm 7$ & \\
\hline Arithmetic magnitude (D) & I. $18 \pm 0.52$ & $0.95 \pm 0.44$ & $0.79 \pm 0.50$ & $1.33 \pm 1.09$ & \\
\hline $95 \% \mathrm{Cl}$ & $0.94-1.43$ & $0.74-1.16$ & $0.56-1.02$ & $0.82-1.83$ & \\
\hline \multicolumn{5}{|c|}{ Postoperative 3 months astigmatism } & 0.0002 \\
\hline Vector magnitude (D) & $1.20 \pm 2.32$ & $0.72 \pm 1.40$ & $0.14 \pm 1.15$ & $0.31 \pm 1.40$ & \\
\hline Vector meridian (degrees) & $53 \pm 26$ & $68 \pm 23$ & $20 \pm 18$ & $21 \pm 10$ & \\
\hline Arithmetic magnitude (D) & $2.21 \pm 1.32$ & $1.4 I \pm 0.63$ & $0.94 \pm 0.64$ & $\mathrm{I} . \mathrm{II} \pm 0.88$ & \\
\hline $95 \% \mathrm{Cl}$ & $1.60-2.83$ & $1.12-1.71$ & $0.64-1.24$ & $0.70-1.52$ & \\
\hline \multicolumn{6}{|l|}{$P$-values ${ }^{\ddagger}$} \\
\hline Against EXP & $0.0068 *$ & - & - & - & \\
\hline Against exLOT & $<0.000$ I** & 0.1052 & - & - & \\
\hline Against $\mu \mathrm{LOT}$ & $0.0003^{*}$ & 0.3001 & 0.5526 & - & \\
\hline \multicolumn{5}{|l|}{ Surgically induced astigmatism } & $<0.0001$ \\
\hline Vector magnitude (D) & $1.01 \pm 2.27$ & $0.62 \pm 1.20$ & $0.23 \pm 1.04$ & $0.12 \pm 0.75$ & \\
\hline Vector meridian (degrees) & $56 \pm 26$ & $74 \pm 24$ & $112 \pm 17$ & $97 \pm 21$ & \\
\hline Arithmetic magnitude (D) & $2.03 \pm 1.37$ & $1.17 \pm 0.64$ & $0.86 \pm 0.59$ & $0.62 \pm 0.42$ & \\
\hline $95 \% \mathrm{Cl}$ & $|.66-2.4|$ & $0.80-1.54$ & $0.49-1.24$ & $0.25-0.99$ & \\
\hline \multicolumn{6}{|l|}{$P$-values ${ }^{\ddagger}$} \\
\hline Against EXP & $0.0017^{*}$ & - & - & - & \\
\hline Against exLOT & $<0.000 \mathrm{I} * *$ & $0.247 \mathrm{I}$ & - & - & \\
\hline Against $\mu \mathrm{LOT}$ & $<0.0001 * *$ & 0.0402 & 0.3599 & - & \\
\hline
\end{tabular}

Notes: $P$-values are calculated for the arithmetic means among the four glaucoma surgeries by one-way analysis of variance $(\#)$ followed by comparison between each pair of groups using the post hoc Student $t$-test $\left(^{\ddagger}\right)$. In the post hoc test, based on Bonferroni's method to correct multiple comparisons, $P<0.0083$ and $P<0.00 I 7$ are considered significant at the probability levels of $5 \%(*)$ and $1 \%(* *)$, respectively. Astigmatism data are expressed as the mean \pm standard deviation.

Abbreviations: LEC, trabeculectomy; EXP, EX-PRESS ${ }^{\circledR}$ shunt; exLOT, ab externo trabeculotomy; $\mu$ LOT, microhook ab externo trabeculotomy; Cl, confidence interval.

while the correlation disappeared 6 months postoperatively. ${ }^{9}$ El-Saied et al did not find a significant association between the astigmatic change and percentage decrease in IOP. ${ }^{8}$ Accordingly, the SIA likely is associated with the low IOP level itself rather than the changes in IOP. ${ }^{8}$ The eye is probably more susceptible to deformation when the IOP is the lowest, and the lower IOPs achieved after filtration surgeries were associated with higher SIA and worse VA in this study. Hugkulstone did not find a significant association between the postoperative changes in corneal radii and the final VA in a small study of LEC $(n=10)$, although the details of the analysis (ie, statistical method used and the conversion or lack of conversion of the VA into logMAR) were unknown. ${ }^{1}$ Our observation seems reasonable because we sometimes see poor VA due to corneal astigmatism in our patients. Our observation also agreed well with a previous observation of early recovery of the BCVA and less variations in the IOP after EXP compared with after LEC. ${ }^{18}$

We created a rectangular scleral flap in the LEC and EXP procedures as reported in previous studies that assessed the postoperative astigmatism. ${ }^{1,2,4-7}$ while we created a triangular flap in the exLOT procedure. Because of the ease of readaptation of the posterior flap edge, a triangular flap might be less associated with the first mechanism than the rectangular flap, but this needs to be tested. As indicated in the descriptions of the surgical procedures, the second scleral flap was excised during the exLOT procedure, which is identical to the lake created during the deep sclerectomy, viscocanalostomy, and nonpenetrating LEC. ${ }^{7}$ Nonpenetrating glaucoma surgery is associated with less SIA than penetrating LEC. ${ }^{7}$ Because the tissue was not perforated under the flap, the second mechanism in the exLOT procedure might not be as significant as in the LEC procedure, thus partly explaining the lower SIA in the exLOT procedure than in the LEC procedure in our study.

The SIA after $\mu$ LOT did not differ greatly from that after microincisional cataract surgery (mean SIA magnitude, $0.42 \mathrm{D}$ after a $1.8 \mathrm{~mm}$ incision coaxial phacoemulsification and $0.5 \mathrm{D}$ after $1.7 \mathrm{~mm}$ incision bimanual phacoemulsification). ${ }^{19}$ Conjunctival and scleral sparing with the ab interno technique, short surgical time, moderate IOP reduction, and no bleb-related complications of $\mu \mathrm{LOT}^{11,12}$ fulfill the conditions of MIGS, ${ }^{20,21}$ as with the recent techniques of ab interno trabeculotomy/LEC and gonio-bypass surgeries such as the 

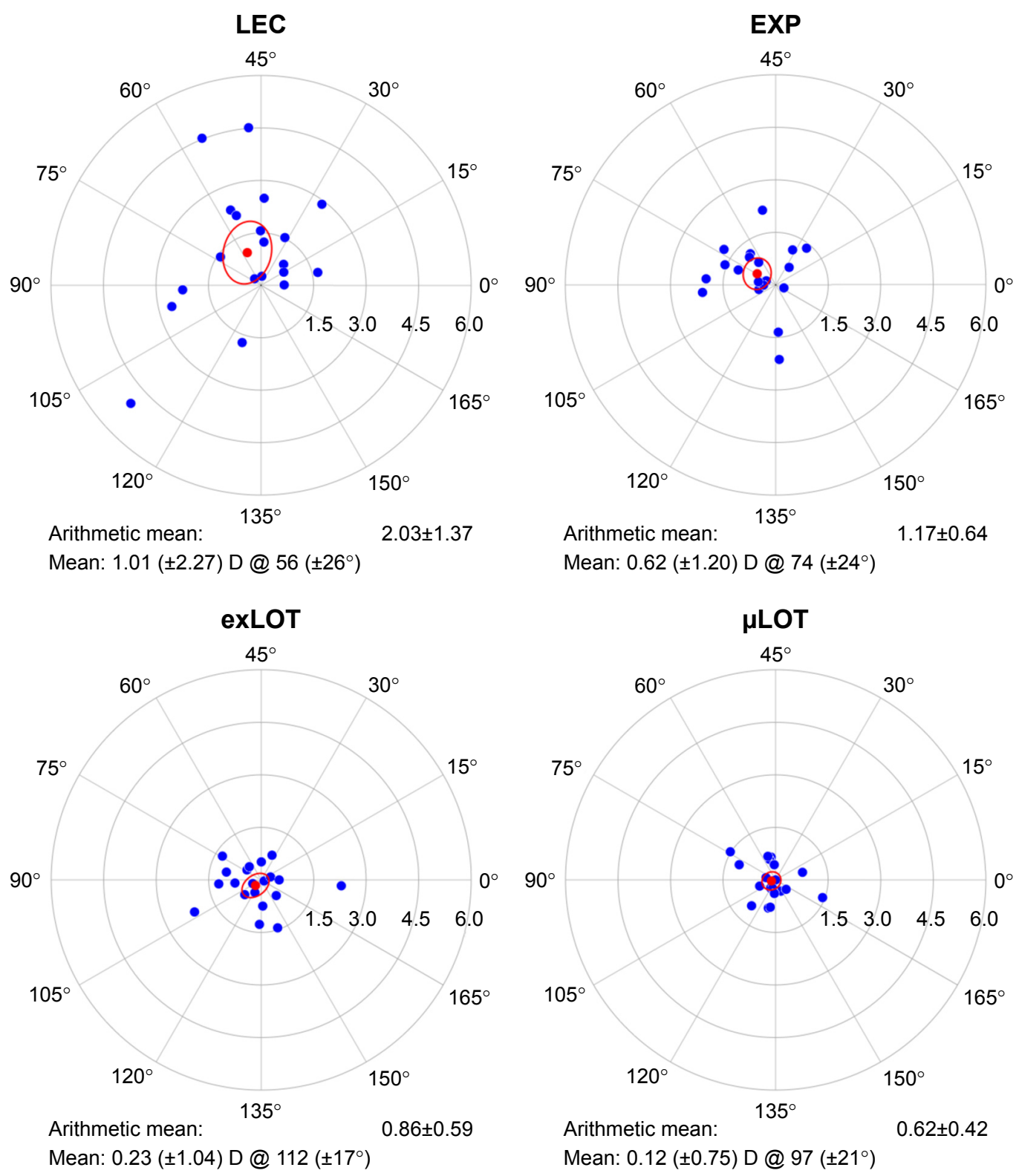

Figure I A scatter plot of SIA in each surgical group. The red and blue dots in each chart indicate the mean SIA vectors of the group and the SIA of each eye, respectively. The red circles indicate $95 \%$ confidence interval. All the astigmatic values are expressed in a plus-cylinder format. The astigmatism in the direction of $0^{\circ}$ indicates against-therule astigmatism, and that in the direction of $90^{\circ}$ indicates with-the-rule astigmatism. Each ring indicates I.5 D.

Abbreviations: SIA, surgically induced astigmatism; LEC, trabeculectomy; EXP, EX-PRESS ${ }^{\circledR}$ shunt; exLOT, ab externo trabeculotomy; $\mu$ LOT, microhook ab interno trabeculotomy.

Table 3 Possible association between SIA magnitude, IOP, and BCVA at 3 months postoperatively $(n=80)$

\begin{tabular}{llll}
\hline Parameters & $\begin{array}{l}\text { SIA } \\
\text { magnitude (D) }\end{array}$ & $\begin{array}{l}\text { BCVA } \\
\text { (logMAR) }\end{array}$ & $\begin{array}{l}\text { IOP } \\
(\mathbf{m m H g})\end{array}$ \\
\hline SIA magnitude (D) & - & $P=0.00 \mid 3^{* *}$ & $P=\left.0.003\right|^{* *}$ \\
BCVA (logMAR) & $r=0.3538$ & - & $P=\left.0.005\right|^{*}$ \\
IOP $(\mathrm{mmHg})$ & $r=-0.3265$ & $r=-0.3105$ & - \\
\hline
\end{tabular}

Notes: The correlation coefficient $(r)$ and $P$-values are calculated by Spearman's rank correlation test between parameters. Based on Bonferroni's method to correct multiple comparisons, $P<0.0167$ and $P<0.0033$ are considered significant at the probability levels of $5 \%(*)$ and $1 \%(* *)$, respectively.

Abbreviations: SIA, surgically induced astigmatism; IOP, intraocular pressure; $B C V A$, best-corrected visual acuity; logMAR, logarithm of the minimum angle of resolution.
Trabectome (NeoMedix Corp., Tustin, CA, USA), ${ }^{22}$ iStent (Glaukos, San Clemente, CA, USA), ${ }^{23}$ gonioscopy-assisted transluminal LOT, ${ }^{24,25}$ canaloplasty, ${ }^{26,27}$ dual-blade LEC (New World Medical Inc., Rancho Cucamonga, CA, USA), ${ }^{21,28}$ and TRAB360 surgery (Sight Sciences Inc., Menlo Park, CA, USA). Other than the two previously described mechanisms of the astigmatism after LEC (ie, surgically induced gape around the scleral flap and creation of unsupported corneal edge), the postulations about the mechanisms of SIA include tissue contraction around the LEC site secondary to extensive scleral cautery, ${ }^{4}$ the wound-healing process 
of the subconjunctiva, ${ }^{5,6}$ and corneal steepening provoked by the pressure of a large drainage bleb under the eyelid. ${ }^{4}$ None of these mechanisms is present in the recent MIGS procedures; accordingly, minimal induction of astigmatism and less astigmatism-related decrease of BCVA observed in the $\mu$ LOT group would also be expected with the other MIGS procedures.

The limitations of the current study included the retrospective study design, analysis of the SIA at one postoperative time point, inclusion of various glaucoma types, inclusion of both phakic and pseudophakic eyes in the four surgical groups, and inclusion of eyes with and without laser suture lysis of the scleral flap in the filtration surgery group. Since the WTR astigmatism seen up to 3 months postoperatively with LEC shifted to against-the-rule astigmatism 6 months postoperatively and later, ${ }^{6,7}$ our observation might change if we assess the SIA at different time points. Since no effects of laser suture lysis ${ }^{3}$ or lens status (phakic or pseudophakic $)^{9}$ on the SIA have been reported previously, we believe that our study design was reasonable to compare the character of the SIA in the early postoperative period after glaucoma surgeries.

\section{Acknowledgments}

The microhooks used in this study were codeveloped by Masaki Tanito and Inami \& Co., Ltd. and were provided by Inami \& Co., Ltd. The authors acknowledge Shiho Kimachi, Jo Fujihara, Shinya Soda, Kaori Ishitobi, and Kumiko Konishi for their support in chart review of the subjects. They also acknowledge the use of the SIA Calculator Version 2.1 (C2010, Dr Saurabh Sawhney, Dr Aashima Aggarwal) in the analysis of data in this study.

\section{Disclosure}

The authors report no conflicts of interest in this work.

\section{References}

1. Hugkulstone CE. Changes in keratometry following trabeculectomy. Br J Ophthalmol. 1991;75:217-218.

2. Cunliffe IA, Dapling RB, West J, et al. A prospective study examining the changes in factors that affect visual acuity following trabeculectomy. Eye. 1992;6:618-622.

3. Rosen WJ, Mannis MJ, Brandt JD. The effect of trabeculectomy on corneal topography. Ophthalmic Surg. 1992;23:395-398.

4. Claridge KG, Galbraith JK, Karmel V, et al. The effect of trabeculectomy on refraction, keratometry and corneal topography. Eye (Lond). 1995; 9(Pt 3):292-298.

5. Hong YJ, Choe CM, Lee YG, et al. The effect of mitomycin-C on postoperative corneal astigmatism in trabeculectomy and a triple procedure. Ophthalmic Surg Lasers. 1998;29(6):484-489.

6. Kook MS, Kim HB, Lee SU. Short-term effect of mitomycin-C augmented trabeculectomy on axial length and corneal astigmatism. J Cataract Refract Surg. 2001;27:518-523.
7. Egrilmez S, Ates H, Nalcaci S, et al. Surgically induced corneal refractive change following glaucoma surgery: nonpenetrating trabecular surgeries versus trabeculectomy. J Cataract Refract Surg. 2004; 30:1232-1239.

8. El-Saied HM, Foad PH, Eldaly MA, et al. Surgically induced astigmatism following glaucoma surgery in Egyptian patients. J Glaucoma. 2014;23(3):190-193.

9. Delbeke H, Stalmans I, Vandewalle E, et al. The effect of trabeculectomy on astigmatism. J Glaucoma. 2016;25(4):e308-e312.

10. Willekens K, Pinto LA, Delbeke H, et al. Trabeculectomy with moorfields conjunctival closure technique offers safety without astigmatism induction. J Glaucoma. 2016;25(5):e531-e535.

11. Tanito M, Sano I, Ikeda Y, et al. Short-term results of microhook ab interno trabeculotomy, a novel minimally invasive glaucoma surgery in Japanese eyes: initial case series. Acta Ophthalmol. 2016;95(5): e354-e360.

12. Tanito M, Sano I, Ikeda Y, Fujihara E. Microhook ab interno trabeculotomy, a novel minimally invasive glaucoma surgery, in eyes with open-angle glaucoma with scleral thinning. Acta Ophthalmol. 2016;94(5):e371-e372.

13. Tanito M, Sano I, Ohira A. A case report of progressive obstruction of Ex-PRESS miniature glaucoma shunt after transient flat anterior chamber and treatment using Nd:YAG laser. BMC Ophthalmol. 2015;15:2.

14. Nonoyama S, Tanito M, Katsube T, et al. Decompression retinopathy and serous retinal detachment after trabeculotomy in a patient with systemic amyloidosis. Jpn J Ophthalmol. 2009;53:73-75.

15. Jauhari N, Chopra D, Chaurasia RK, et al. Comparison of surgically induced astigmatism in various incisions in manual small incision cataract surgery. Int J Ophthalmol. 2014;7(6):1001-1004.

16. Alpins NA. A new method of analyzing vectors for changes in astigmatism. J Cataract Refract Surg. 1993;19:524-533.

17. Holladay JT, Moran JR, Kezirian GM. Analysis of aggregate surgically induced refractive change, prediction error, and intraocular astigmatism. J Cataract Refract Surg. 2001;27(1):61-79.

18. Netland PA, Sarkisian SR, Moster MR, et al. Randomized, prospective, comparative trial of EX-PRESS glaucoma filtration device versus trabeculectomy (XVT study). Am J Ophthalmol. 2014;157:433-440.e3.

19. Wilczynski M, Supady E, Piotr L, et al. Comparison of surgically induced astigmatism after coaxial phacoemulsification through $1.8 \mathrm{~mm}$ microincision and bimanual phacoemulsification through $1.7 \mathrm{~mm}$ microincision. J Cataract Refract Surg. 2009;35(9):1563-1569.

20. Ahmed II. MIGS and the FDA: what's in a name? Ophthalmology. 2015;122:1737-1739.

21. Kahook MY, Seibold LK, SooHoo JR, et al. A nuanced approach to the surgical management of glaucoma. Middle East Afr J Ophthalmol. 2015;22(1):1.

22. Minckler DS, Baerveldt G, Alfaro MR, et al. Clinical results with the Trabectome for treatment of open-angle glaucoma. Ophthalmology. 2005;112(9):962-967.

23. Malvankar-Mehta MS, Chen YN, Iordanous Y, et al. iStent as a solo procedure for glaucoma patients: a systematic review and meta-analysis. PLoS One. 2015;10(5):e0128146.

24. Grover DS, Godfrey DG, Smith O, et al. Gonioscopy-assisted transluminal trabeculotomy, ab interno trabeculotomy: technique report and preliminary results. Ophthalmology. 2014;121(4):855-861.

25. Sato T, Hirata A, Mizoguchi T. Prospective, noncomparative, nonrandomized case study of short-term outcomes of 360 degrees suture trabeculotomy ab interno in patients with open-angle glaucoma. Clin Ophthalmol. 2015;9:63-68.

26. Khaimi MA. Canaloplasty: a minimally invasive and maximally effective glaucoma treatment. J Ophthalmol. 2015;2015:485065.

27. Matlach J, Dhillon C, Hain J, et al. Trabeculectomy versus canaloplasty (TVC study) in the treatment of patients with open-angle glaucoma: a prospective randomized clinical trial. Acta Ophthalmol. 2015;93(8): $753-761$.

28. Seibold LK, Soohoo JR, Ammar DA, et al. Preclinical investigation of ab interno trabeculectomy using a novel dual-blade device. Am J Ophthalmol. 2013;155:524-529. 


\section{Publish your work in this journal}

Clinical Ophthalmology is an international, peer-reviewed journal covering all subspecialties within ophthalmology. Key topics include: Optometry; Visual science; Pharmacology and drug therapy in eye diseases; Basic Sciences; Primary and Secondary eye care; Patient Safety and Quality of Care Improvements. This journal is indexed on
PubMed Central and CAS, and is the official journal of The Society of Clinical Ophthalmology (SCO). The manuscript management system is completely online and includes a very quick and fair peer-review system, which is all easy to use. Visit http://www.dovepress.com/ testimonials.php to read real quotes from published authors. 